\title{
Turbulence Decay in Stopped Flow Experiments by Measurements of Electric Dichroism
}

\author{
Dietmar Porschke \\ Max Planck Institut für Biophysikalische Chemie, D 37077 Göttingen, Germany \\ Received: September 13, 1999; In Final Form: November 9, 1999
}

\begin{abstract}
Various macromolecules of widely different chemistry and structure show unusual rotational diffusion time constants in stopped flow experiments. These effects have been characterized by measurements of electric dichroism in a combined stopped-flow-electric-field-jump instrument. DNA restriction fragments in the range of chain lengths up to 179 base pairs do not show anything unusual yet: the dichroism decay times correspond to the standard values reflecting unperturbed rotational diffusion. For fragments with more than 200 base pairs, the dichroism measured directly after stop of the flow revealed decay time constants clearly smaller than the corresponding values found without flow. The reduction of the time constants for overall rotational diffusion is $\sim 10 \%$ for a fragment with $256 \mathrm{bp}$ and increases to $\sim 70 \%$ for DNA with $800 \mathrm{bp}$. The decay time constants return to the usual values within a few $\mathrm{ms}$ after stop of flow. The limiting electric dichroism of the flow induced state is virtually identical with that of standard DNA and, thus, the local secondary structure remains in the B-form. However, the polarizability of the flow induced state is clearly reduced. These results seem to indicate a flexible state of DNA induced by shear stress, resulting from breaking of stacking interactions. The kinetics can be described by a model with parallel reactions leading to the native state with a time constant of $\sim 1 \mathrm{~ms}$. Effects observed for single stranded poly(A) are virtually identical to those of double stranded DNA for samples of corresponding hydrodynamic dimensions. The time constants for the transition to the standard state are again $\sim 1 \mathrm{~ms}$, although stacking reactions of poly(A) occur in the time range $\leq 1 \mu \mathrm{s}$. Further experiments with other macromolecules or macromolecular aggregates indicate that acceleration of rotational motion is a general phenomenon under stopped flow conditions: for example bacteriorhodopsin membrane disks and colloidal suspensions of the fibrous claylike silicate attapulgite show very high accelerations of their rotational diffusion time constants after stopped flow by factors of about 3000 . The return to the usual state both for bacteriorhodopsin and for attapulgite extends over a broad time range up to $\sim 100 \mathrm{~ms}$. Although it cannot be excluded that these effects are partly due to kinking of the structures, the similarity of the data for materials of very different structures indicates that the acceleration of rotational diffusion is mainly due to the existence of turbulence. The rotational motion of small molecules, such as 95 bp DNA, is much faster than rotational motion of corresponding volume elements of the solvent and, thus, these molecules do not indicate turbulence. The data demonstrate that turbulence decays over a broad time range: small vortices with high rates of rotational motion decay first, merge into larger ones with lower rotation rates, until finally the largest ones compatible with the dimension of the observation chamber are reflected only by particles with relatively large dimensions and slow rotational diffusion. These results demonstrate the mode of turbulence in stopped flow observation cells, the scaling of its decay, and the utility of electrooptical techniques for the characterization of turbulence.
\end{abstract}

\section{Introduction}

The stopped flow technique is widely used for the characterization of reactions ${ }^{1,2}$ in the time range down to $\sim 1 \mathrm{~ms}$. The experimental setup is relatively simple and commercially available, the demand for the amount of material is moderate and, thus, it is very popular among biochemists for the characterization of biochemical reactions, including those of macromolecular components. Observation of reactions at a high time resolution requires fast mixing of components to homogeneous solutions by turbulent flow in a mixing chamber. Usually it is assumed that there is no turbulence anymore in the observation chamber.

The present investigation has not been started with the goal to characterize turbulence in stopped flow experiments. During measurements of various DNA samples using a recently developed stopped-flow-field-jump instrument ${ }^{3}$ unusual effects

\footnotetext{
* E-Mail: dpoersc@gwdg.de. Fax: 49-551-2011168.
}

were found for rotational diffusion detected by the decay of electric dichroism. ${ }^{4}$ These effects were first assigned to kinking of DNA double helices, until measurements on different nucleic acid samples indicated that an interpretation by kinking raises some problems. Further extension of the set of compounds used in the investigation finally indicated that the effects are most likely caused by a general hydrodynamic phenomenon. The observed effects show a special scaling with the hydrodynamic dimensions of the macromolecules, which indicates that all effects are directly due to turbulence. The results illustrate the existence of scaling rules for the dimensions and time constants of vortices in decaying turbulence under stopped flow conditions. These results also show that the electric dichroism method can be very useful for the characterization of turbulence.

\section{Materials and Methods}

A DNA fragment with 95 base pairs and 4 single stranded residues at each end was prepared from plasmid pRW574 
(kindly provided by W. Hillen) by digestion with EcoRI restriction endonuclease. The fragment was isolated by Sepharose 4B column chromatography. Digestion of the plasmid DNA by HaeIII restriction endonuclease provided a mixture of fragments, including fragments with 179, 256, "440" (actually a mixture of fragments, see below) and 859 base pairs, which were separated by ion exchange HPLC. The longest fragment resulting from HaeIII digestion (998 base pairs) was isolated and digested further by EcoRI; subsequent separation by ion exchange HPLC provided a fragment with 359 base pairs and 4 single stranded residues at one end. The sample with " 440 " base pairs contained a mixture of fragments with 413, 436, 445, and 446, base pairs, which were not separated. DNA samples with distributions of chain lengths and sequences but having blunt ends were prepared from calf thymus DNA by HaeIII + AluI digestion and separated by Sepharose 4B column chromatography. The average chain length of these DNA samples was determined by comparison with standard fragments on polyacrylamide gel electrophoresis. All DNA samples were dialyzed extensively first against $1 \mathrm{M} \mathrm{NaCl}, 10 \mathrm{mM} \mathrm{Na}$ cacodylate pH 7.0, $1 \mathrm{mM}$ EDTA and finally against $1 \mathrm{mM} \mathrm{NaCl}$, $1 \mathrm{mM}$ Na-cacodylate pH 7.0, $0.2 \mathrm{mM}$ EDTA (buffer A) or against $1 \mathrm{mM} \mathrm{NaCl}, 1 \mathrm{mM}$ Na-cacodylate $\mathrm{pH} 7.0,100 \mu \mathrm{M}$ $\mathrm{MgCl}_{2}$ (buffer B).

Poly(A) was from Boehringer Mannheim (Germany); 2 samples (obtained at different times) showed very different molecular weight; according to their sedimentation coefficients determined by analytical ultracentrifugation in a Beckman XL-A (5.17 and 8.29 $\mathrm{S}$ at $20^{\circ} \mathrm{C}$ and $0.1 \mathrm{M}$ ionic strength), the average chain lengths are $\sim 490$ and $\sim 1290$ nucleotide residues using the data of Fresco and Doty ${ }^{5}$ for calibration.

Bacteriorhodopsin was kindly provided by the laboratory of Prof. D. Oesterhelt. The attapulgite sample was kindly donated by Dr. R. Oschmann from Dr. Willmar Schwabe GmbH \& Co., Karlsruhe. A suspension was prepared by sonication of attapulgite in buffer A; after sedimentation under gravity at room temperature for 4 days the supernatant was used for measurements.

The stopped-flow-electric-field-jump instrument has been described. ${ }^{3}$ Cells with optical path lengths of 2 and $10 \mathrm{~mm}$ were used for the measurements. One of the factors which determines the time resolution of stopped flow instruments, is the flow rate used for the mixing of reactants. In the present instrument a total volume of $\sim 160 \mu \mathrm{L}$ is mixed together within a total flow time of $\sim 5 \mathrm{~ms}$ during standard stopped flow experiments. Thus, the average flow rate in the cuvette used for the measurements is $32 \mathrm{~mL} / \mathrm{s}$. The sectional area of both cuvettes is $2 \times 2 \mathrm{~mm}^{2}$ and, thus, according to the Reynolds criterion the flow is turbulent not only in the mixing chamber, but also in the measuring cuvette (Reynolds number $\sim 1.5 \times 10^{4}$ ).

After the flow is stopped the state of the solution is analyzed by application of electric field pulses with pulse durations of, e.g., $10 \mu$ s for DNA molecules and $170 \mu$ s for bacteriorhodopsin. These pulses induce partial orientation of the molecules, which is analyzed by measurement of the linear dichroism. The decay of the linear dichroism after pulse termination provides information about rotational diffusion/motion. The electric field pulses are applied at various times $\Delta t$ after the flow is stopped and, thus, the time course of any reaction or change proceeding in the solution after the flow is stopped can be recorded at high sensitivity. The dichroism was measured with the plane of polarized light adjusted parallel to the field vector. Values of the reduced dichroism are given as $\xi=1.5 \times \Delta A_{\|}$, where $\Delta A_{\|}$ is the field induced change of absorbance of light polarized parallel to the field vector and $\bar{A}$ is the isotropic absorbance.

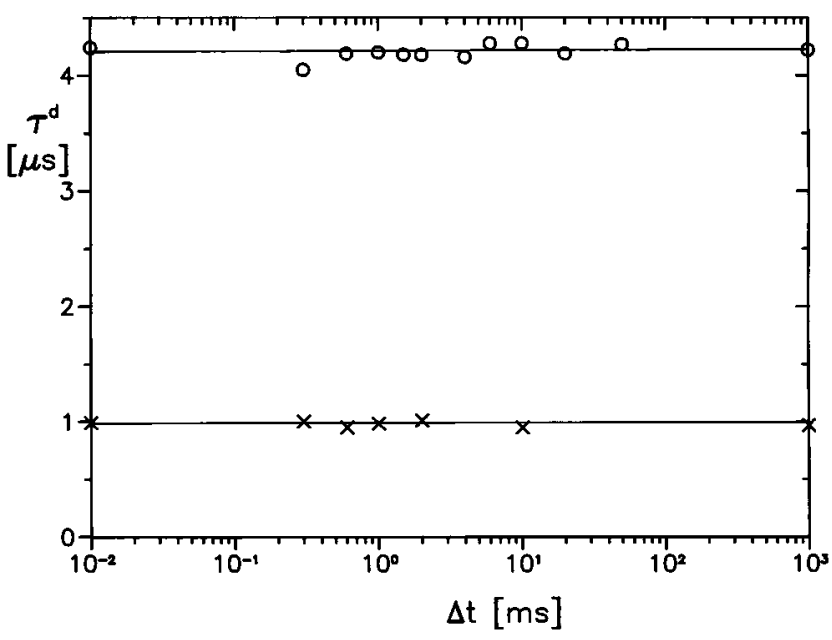

Figure 1. Dichroism decay time constants $\tau^{\mathrm{d}}$ of DNA fragments with $95 \mathrm{bp}(\times)$ and with $179 \mathrm{bp}(\bigcirc)$ measured at different delay times $\Delta t$ after stop of the flow $\left(10^{\circ} \mathrm{C}\right.$, buffer $\left.\mathrm{A}, 248 \mathrm{~nm}\right)$. The $\tau^{\mathrm{d}}$-values given for the $95 \mathrm{bp}$ fragment were obtained by single exponential fits. Double exponential fits were required for the decay curves of the $179 \mathrm{bp}$ fragment; the time constants of the slow relaxation process $\tau_{2}^{\mathrm{d}}$, representing overall rotational diffusion, are shown. The time constants measured at $\Delta t=0$ are shown in this plot at $\Delta t=0.01$.

\section{Results}

Flow Induced Change of DNA Dichroism Decay and its Dependence on DNA Chain Length. Stopped-flow-field-jump experiments on solutions of a $95 \mathrm{bp}$ fragment and recording its electric dichroism at various times after the flow is stopped did not indicate the existence of some special effect. The dichroism decay time constants $\tau_{d}$ are independent of the delay times after the flow is stopped (cf. Figure 1). A similar result was obtained for a fragment with $179 \mathrm{bp}$, although in this case a small reduction of its dichroism decay time constant measured directly after the flow is stopped can be detected (Figure 1). Clear evidence for the existence of some special process was obtained for longer DNA fragments. For example, a DNA fragment with 256 bp showed a clear reduction of its dichroism decay time constant when this time constant was measured directly after the flow was stopped. When the same solution was analyzed again by its electric dichroism at long times after the flow, the decay time constant was back to the well-known standard value. Measurements of the dichroism decay at various delay times after the flow is stopped showed that the dichroism decay time constants return to the standard values within a few milliseconds after the flow is stopped (Figure 2). Corresponding effects were observed for many different DNA samples in the range of chain lengths above 200 base pairs up to 859 base pairs. The decrease of the decay time constant induced by the flow increases with the chain length. A direct comparison of dichroism decay curves measured at 0.4 and $100 \mathrm{~ms}$ after the flow is stopped for a fragment with 859 base pairs is shown in Figure 3. The decay curves for this fragment require three exponential processes for satisfactory fits. The time constants of all the three processes are reduced in decay curves measured at short times after the flow is stopped (cf. details in legend to Figure 3). The dichroism decay curves observed at times $>10 \mathrm{~ms}$ after the flow cannot be distinguished from those measured for samples that have not been subjected to fast flow. Thus, the perturbation induced by fast flow under the conditions of the present experiments is reversible. This is confirmed by gel electrophoresis: the mobility of fragments exposed to stopped flow experiments is identical to the mobility of native fragments. Furthermore, the melting transitions of the DNA fragments used in the present investiga- 


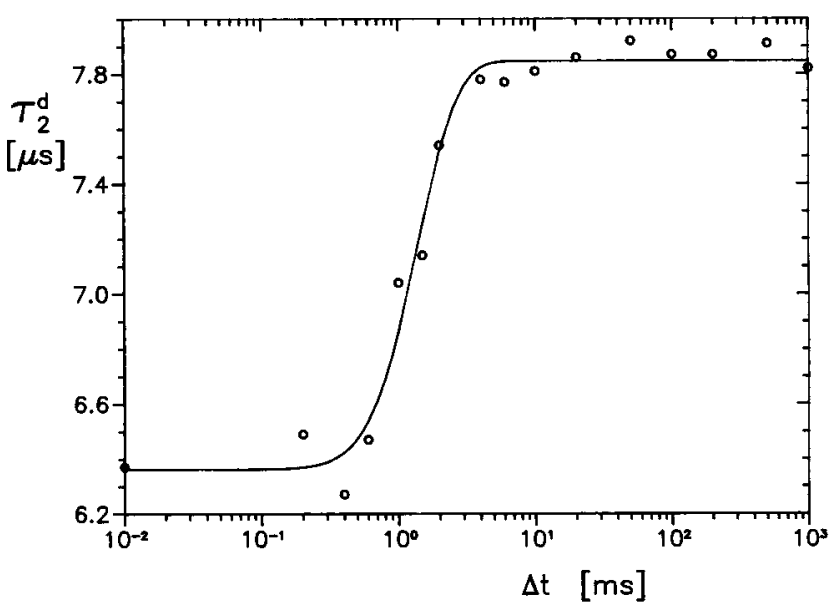

Figure 2. Dichroism decay time constants $\tau_{2, \text { obs }}^{\mathrm{d}}$ of a DNA fragment with $256 \mathrm{bp}$ as a functon of the time $\Delta t$ after stop of the flow (buffer $\mathrm{B}, 10^{\circ} \mathrm{C}, 248 \mathrm{~nm}$ ). The line represents a least-squares fit according to the parallel mechanism with the parameters $\tau_{2, \mathrm{k}}^{\mathrm{d}}=6.36 \mu \mathrm{s}, \tau_{2, \mathrm{n}}^{\mathrm{d}}=7.85$ $\mu \mathrm{s}, \tau_{\mathrm{p}}=0.74 \mathrm{~ms}$, and $\kappa=3.6$. The time constant measured at $\Delta t=0$ is shown in this plot at $\Delta t=0.01$.

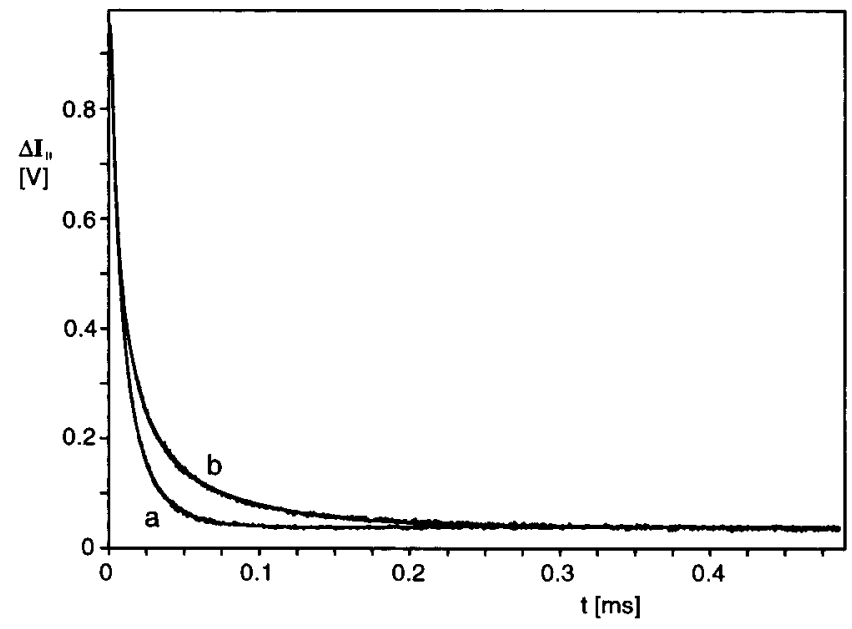

Figure 3. Comparison of the dichroism decay curves obtained for the DNA fragment with 859 base pairs (a) at $0.4 \mathrm{~ms}$ after stop of the flow and (b) at $100 \mathrm{~ms}$ after stop of the flow (buffer B, $10{ }^{\circ} \mathrm{C}, 28.5 \mu \mathrm{M}$ nucleotide residues, electric field pulse $17.8 \mathrm{kV} / \mathrm{cm}$ for $22 \mu \mathrm{s}, 248 \mathrm{~nm}$ ). Least squares fits of the curves provided the following time constants (the numbers in brackets are the amplitudes in percent of the total amplitude; the total amplitudes are 913.4 and $910.5 \mathrm{mV}$ for (a) and (b), respectively): (a) $\tau_{1}^{\mathrm{d}}=1.08 \mu \mathrm{s}$ (15.6), $\tau_{2}^{\mathrm{d}}=5.83 \mu \mathrm{s}$ (39.3), $\tau_{3}^{\mathrm{d}}=$ $17.1 \mu \mathrm{s}$ (45.1); (b) $\tau_{1}^{\mathrm{d}}=2.15 \mu \mathrm{s}$ (34.4), $\tau_{2}^{\mathrm{d}}=13.3 \mu \mathrm{s}(43.7), \tau_{3}^{\mathrm{d}}=61.3$ $\mu \mathrm{s}(22.0)$.

tion are not affected by exposure to the stopped flow field jump procedure: both $T_{m}$ values and the shapes of the transitions are identical before and after exposure within the limits of accuracy.

Measurements of dichroism transients at different times after the flow is stopped clearly demonstrate a special type of kinetics for the return of DNA to its standard state. As shown in Figure 4 for the example of a fragment with 859 base pairs, the decay time constants return to their usual values in a narrow time range between 1 and $10 \mathrm{~ms}$. The plot of the data with a logarithmic time scale leads to a sigmoidal appearance also for simple standard reactions, e.g., of first order. However, in the present case the transition is observed in a relatively narrow time range, indicating a special type of mechanism (cf. next section).

Measurements of the electric dichroism can be used to get more information about structures in solution by evaluation of

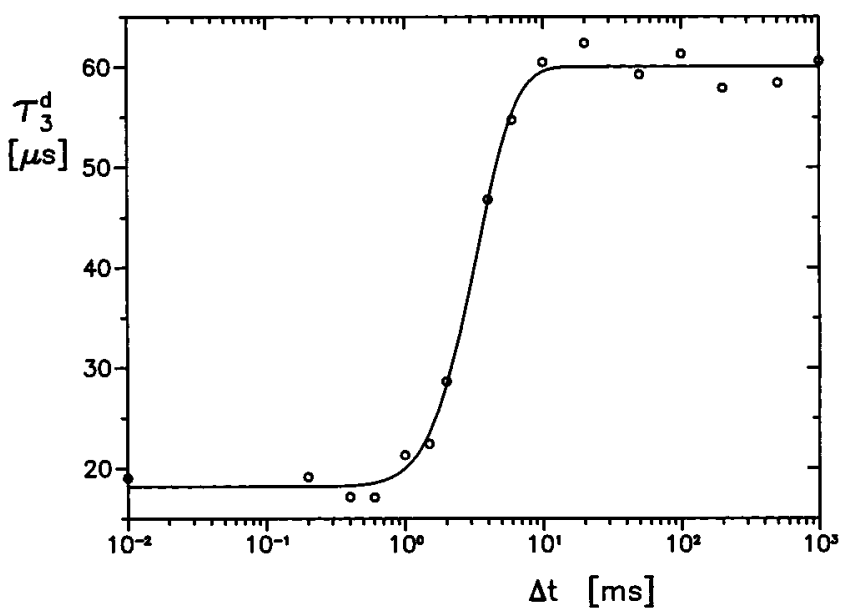

Figure 4. Dichroism decay time constant $\tau_{3 \text {,obs }}^{\mathrm{d}}$ of a DNA fragment with $859 \mathrm{bp}$ as a function of the time $\Delta t$ after stop of the flow (buffer $\left.\mathrm{B}, 10{ }^{\circ} \mathrm{C}, 248 \mathrm{~nm}\right)$. The line represents a least-squares fit according to the parallel mechanism with the parameters $\tau_{3, \mathrm{k}}^{\mathrm{d}}=18.2 \mu \mathrm{s}, \tau_{3, \mathrm{n}}^{\mathrm{d}}=60.0$ $\mu \mathrm{s}, \tau_{\mathrm{p}}=1.7 \mathrm{~ms}$, and $\kappa=3.8$. The time constant measured at $\Delta t=0$ is shown in this plot at $\Delta t=0.01$.

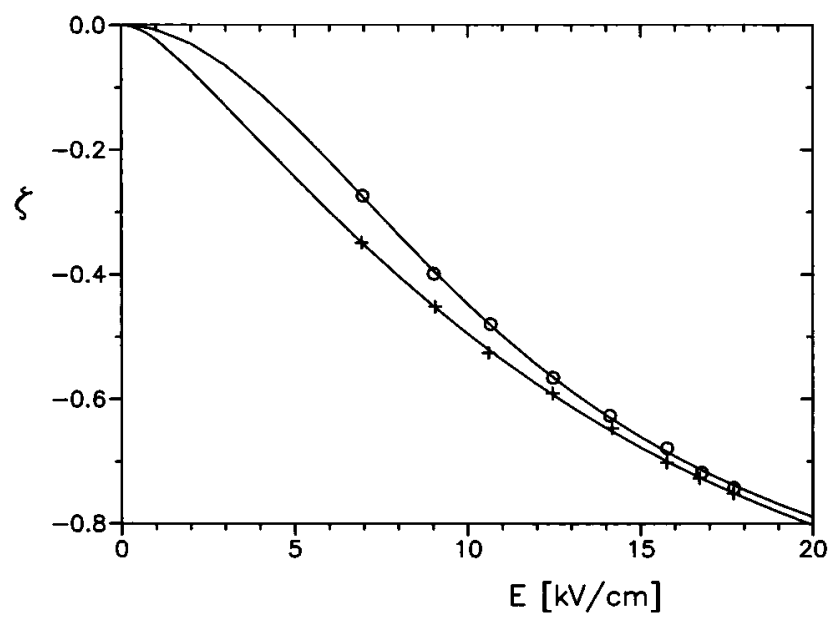

Figure 5. Stationary values of the electric dichroism $\xi$ for a DNA fragment with 859 base pairs at different electric field strengths $E$ for the flow induced state measured directly after stop of the flow $(O)$ and for the native state $(+)$ measured at "infinite" time after stop of the flow (buffer B, $10{ }^{\circ} \mathrm{C}, 248 \mathrm{~nm}$ ). The continuous lines represent least squares fits by the orientation function with saturation of the polarizability ${ }^{6,7}$ with the following parameters for the flow induced state and the native state, respectively: limiting value of the electric dichroism -1.216 and -1.315 ; polarizability $3.72 \times 10^{-32}$ and $11.9 \times 10^{-32} \mathrm{C}$ $\mathrm{m}^{2} \mathrm{~V}^{-1}$; saturating electric field strength 4.05 and $1.07 \mathrm{kV} / \mathrm{cm}$.

stationary values of the dichroism and their dependence on the electric field strength. A comparison of these stationary values at different field strengths is shown in Figure 5 for the state of the 859 DNA fragment immediately after stop of the flow and for the native state at long times after the flow. At low field strengths the stationary dichroism is clearly lower for the flow induced state than for the standard state, whereas at higher field strengths the difference is relatively small. These data have been analyzed by an orientation function with saturation of the polarizability: ${ }^{6,7}$ the polarizability of the field induced state is lower by a factor of $\sim 3$ and the saturation field strength is higher by a factor of $\sim 4$ than those of the native state; the limit value of the electric dichroism of the flow induced and the standard state are equivalent within experimental accuracy and correspond to standard values for DNA fragments in the literature ${ }^{6}$ (cf. fitted values in legend to Figure 5). Similar results were obtained for the restriction fragments with 256,359 , and " 440 " base pairs. 
These data indicate that the local structure of the DNA is virtually the same for both states, whereas the long-range structure appears to be different. The decrease of the polarizability and the increase of the saturation field strength for the flow induced state suggest reduced segment lengths for polarization. ${ }^{8}$ Thus, the conclusion obtained from the electric parameters is consistent with that from the time constants of overall rotational diffusion.

Dependence on AT/GC Content, Temperature, and Ion Concentration. It may be expected that the properties of DNA double helices characterized by the experiments described above are determined by the base sequence and, thus, it should be useful to do corresponding experiments on DNA double helices with a single type of base pair. These polymers, like poly[d(AT)] and poly[d(GC)], are available only in the form of relatively broad distributions of chain lengths. For this reason the dichroism decay time constants cannot be defined as precisely as for restriction fragments. Nevertheless, these polymers were used for stopped flow field jump experiments for a first test. Both poly[d(AT)] and poly[d(GC)] with chain lengths in the range around 1000 base pairs showed a clear flow induced decrease of their dichroism decay time constants. In both cases the decay time constants returned to the standard values within a few milliseconds; the kinetics of the transition from the perturbed to the native state is equivalent to that described above within the limits of experimental accuracy.

Some dependences of the flow induced effect were tested with DNA samples containing mixed sequences and chain length distributions generated by enzymatic digestion (cf. Materials and Methods Section). Measurements on a sample with an average of 800 base pairs showed that the flow induced reduction of the decay time constant is very similar in the buffers $\mathrm{A}$ and $\mathrm{B}$. Corresponding results were obtained for restriction fragments. Thus, the presence or absence of $\mathrm{Mg}^{2+}$ ions does not have any strong influence on the effect. Measurements on a sample with an average of 650 base pairs in buffer $A$ at 2, 10 , and $20{ }^{\circ} \mathrm{C}$ showed only a minor dependence on the temperature. According to these data the activation enthalpy for the rate of the resealing reaction is relatively low (in the range $\sim 5 \mathrm{kcal} / \mathrm{mol}$ ). The results demonstrate that both the dependence on the ion composition and on the temperature are certainly not extreme. Future experiments on the temperature dependence should be conducted with restriction fragments, to get results at a higher accuracy.

Model of a Resealing Reaction Mechanism. The experimental data described above for the return from the flow induced state to the standard state do not follow a simple first order reaction mechanism. Deviations from a simple first order rate law may be explained by many different mechanisms. In the present case, the nature of the reaction and the experimental observations suggest simple limit cases of reaction mechanisms. It may be expected that shear stress generates kinks in double helices. If there is more than a single kink per DNA chain, these kinks may be resealed independently and parallel to each other.

In the most simple limit case, resealing of each individual kink may be described by a simple intramolecular reaction

$$
A \underset{k^{-}}{\stackrel{k^{+}}{\rightleftharpoons}} B
$$

with a relaxation time constant

$$
1 / \tau_{\mathrm{p}}=k^{+}+k^{-}
$$

For each individual kink the probability of being resealed is given by

$$
P_{\mathrm{i}}=\left(1-\exp \left(-t / \tau_{\mathrm{p}}\right)\right)
$$

When there are $\kappa$ different kinks in a single chain, the probability that all of them are resealed and the original state of the chain is restored is then given by

$$
P_{\mathrm{c}}=\left(1-\exp \left(-t / \tau_{\mathrm{p}}\right)\right)^{\kappa}
$$

provided that all the different kinks reseal with identical kinetic parameters.

In the present investigation the resealing reaction is observed by recording time constants of rotational diffusion. It is expected that introduction of each kink leads to a reduction of the overall rotational diffusion time constant and, thus, reconstitution of the native state requires resealing of all kinks. However, it is also expected that resealing of each individual kink leads to some increase of the rotational diffusion time constant, even if there are more kinks in the same chain, which are not resealed yet. A quantitative description of this process requires a model that includes the different states of the reaction, which are expected to be in a relatively wide distribution. Such a model should also include an appropriate weighting of the kinking probability, which appears to be particularly high at the center of the polymer chain under the conditions of the present experiments. Another problem is the detection of the various states with different numbers of kinks distributed along the chain. In the experiments described above the distribution of different states has not been resolved. The measurements provide average dichroism decay time constants, which may be used for calculation of distributions, provided that an appropriate model is developed.

A simple limit case is an all or none description, assuming that the dichroism decay time constant of the kinked polymer remains at a low value, until all kinks are resealed. According to this model the dichroism decay time constant of the native state is restored when the last kink is resealed. In the experiments, the relaxation processes representing the limit states of the reaction have not been separated during the reaction and, thus, the observed dichroism decay time constants are average values. These average data may be modeled by the approximation that the dichroism decay time constants measured during the reaction simply follows the probability that all kinks in a chain are resealed (eq 4) according to

$$
\tau_{\mathrm{obs}}^{\mathrm{d}}=\tau_{\mathrm{k}}^{\mathrm{d}}+\left(\tau_{\mathrm{n}}^{\mathrm{d}}-\tau_{\mathrm{k}}^{\mathrm{d}}\right)\left(1-\exp \left(-t / \tau_{\mathrm{p}}\right)\right)^{\kappa}
$$

where $\tau_{\mathrm{obs}}^{\mathrm{d}}$ is the observed dichroism decay time constant; $\tau_{\mathrm{k}}^{\mathrm{d}}$ and $\tau_{\mathrm{n}}^{\mathrm{d}}$ are the dichroism decay time constants of the kinked and the native state, respectively.

The parameter $\kappa$ determines the width of the reaction progress curves. When $\kappa$ is increased, the width of the transition on the time scale is reduced considerably: for $\kappa=1$ it takes an interval of about 1.34 units on the logarithmic time scale to go from 10 to $90 \%$ of the transition, whereas at $\kappa=10$ this interval is reduced to about 0.26 units (corresponding to factors of $\sim 21.9$ and $\sim 1.8$, respectively, on a linear time scale).

Sets of experimental data, such as those shown in Figures 2 and 4, were analyzed in terms of the model discussed above. Least-squares fits demonstrate that the model can be used to describe the available experimental data at a satisfactory accuracy. For illustration, the decay time constants for the limit state of the reaction have been interpreted in terms of effective chain lengths on the basis of the wormlike chain model using 
TABLE 1: Parameters obtained from Dichroism Decay Time Constants Measured at Different Times after the Flow Was Stopped by the Parallel Resealing Model $^{a}$

\begin{tabular}{cccccccc}
\hline DNA [bp $]$ & $n$ & $\tau_{\mathrm{p}}[\mathrm{ms}]$ & $\kappa$ & $\tau_{\mathrm{k}}^{\mathrm{d}}[\mu \mathrm{s}]$ & $\tau_{\mathrm{n}}^{\mathrm{d}}[\mu \mathrm{s}]$ & $N_{\mathrm{k}}^{\mathrm{eff}}[\mathrm{bp}]$ & $N_{\mathrm{n}}^{\text {eff }}[\mathrm{bp}]$ \\
\hline 256 & 2 & 0.7 & 3.6 & 6.36 & 7.85 & 229 & 252 \\
359 & 2 & 1.1 & 2.4 & 9.13 & 13.9 & 270 & 328 \\
“440" & 3 & 1.0 & 5.5 & 13.3 & 23.0 & 320 & 415 \\
859 & 3 & 1.7 & 3.8 & 18.2 & 60.0 & 370 & 670
\end{tabular}

${ }^{a} n$ number of exponentials used for fitting of the dichroism decay curves; $\tau_{\mathrm{p}}$ reaction time constant of the parallel model; $\kappa$ number of parallel processes according to the model; $\tau_{\mathrm{k}}^{\mathrm{d}}$ and $\tau_{\mathrm{n}}^{\mathrm{d}}$ dichroism decay time constants of the kinked and the native state, respectively; $N_{\mathrm{k}}^{\text {eff }}$ and $N_{\mathrm{n}}^{\text {eff }}$ effective number of base pairs according to the wormlike chain model (cf. text) for the kinked and the native state, respectively. Buffer B, $10{ }^{\circ} \mathrm{C}$; estimated accuracy; $\tau_{\mathrm{p}} \pm 20 \% ; \kappa \pm 1 ; \tau_{\mathrm{k}}^{\mathrm{d}}$ and $\tau_{\mathrm{n}}^{\mathrm{d}} \pm 3 \%$.

standard values for the persistence length ${ }^{9,10}(50 \mathrm{~nm})$, the effective hydrodynamic diameter of the double helix $(2.5 \mathrm{~nm})$, the rise per base pair $(0.34 \mathrm{~nm})$ and the viscosity $\left(1.30710^{-3}\right.$ $\mathrm{kg} \mathrm{m}^{-1} \mathrm{~s}^{-1}$ for water at $\left.10^{\circ} \mathrm{C}\right)$. The wormlike chain model was used in a combination ${ }^{10}$ of the parameters for rigid rods by Tirado et al. ${ }^{11}$ with factors for wormlike chains derived by a Monte Carlo procedure. ${ }^{12}$ In the flow induced state, these effective chain lengths are much lower than the contour lengths of the DNA double helices (cf. Table 1).

According to the parallel reaction model it may be expected that the number of kinks $\kappa$ increases with increasing chain length. The fitted $\kappa$ values are quite sensitive to details of the data set. Due to this sensitivity and the given level of accuracy of the experimental data, it is not possible to present a definite conclusion on the chain length dependence of $\kappa$. If the time constants are determined by some activation barrier of a conformational transition, it should be expected that the reaction time constant of the parallel model $\tau_{\mathrm{p}}$ is independent of the chain length; the experimental data indicate an increase of $\tau_{\mathrm{p}}$ with increasing chain length (cf. Table 1). Some difference between expectation and experimental results may be attributed to simplifications used in the present form of the parallel reaction model.

It should be noted that the experimental data may also be described with reasonable accuracy by a sequential reaction mechanism of the type $\mathrm{A} \rightarrow \mathrm{B} \rightarrow \mathrm{C}$, for example. Results of this description are not shown, because the final analysis of all the available data does not support an interpretation in terms of a reaction mechanism.

Single stranded poly(A): Similar to Double Stranded DNA. For comparison, the experiments described above for various DNA samples were repeated with two poly(A) samples of different chain length. A sample with $\sim 490$ adenylate residues showed a dichroism decay curve which required two exponentials for satisfactory fitting. The time constant reflecting overall rotational diffusion was $6.6 \mu \mathrm{s}$ under standard conditions and was accelerated to $5.0 \mu \mathrm{s}$ immediately after the flow was stopped. The dichroism decay curves of the second sample with 1290 adenylate residues required three exponentials for fitting. The time constant of the third exponential, reflecting overall rotational diffusion, was $87 \mu$ s under standard conditions and was accelerated to $23.1 \mu \mathrm{s}$ immediately after the flow was stopped. The time course of the transition from the flow induced state to the standard state is shown in Figure 6. The data can be fitted at a high accuracy by the parallel reaction model (equation 5) with a reaction time constant of $0.9 \mathrm{~ms}$.

The persistence length of single stranded poly(A) is lower than that of double stranded DNA. ${ }^{10,13,14}$ Because of this difference the overall rotational time constant of a poly(A)

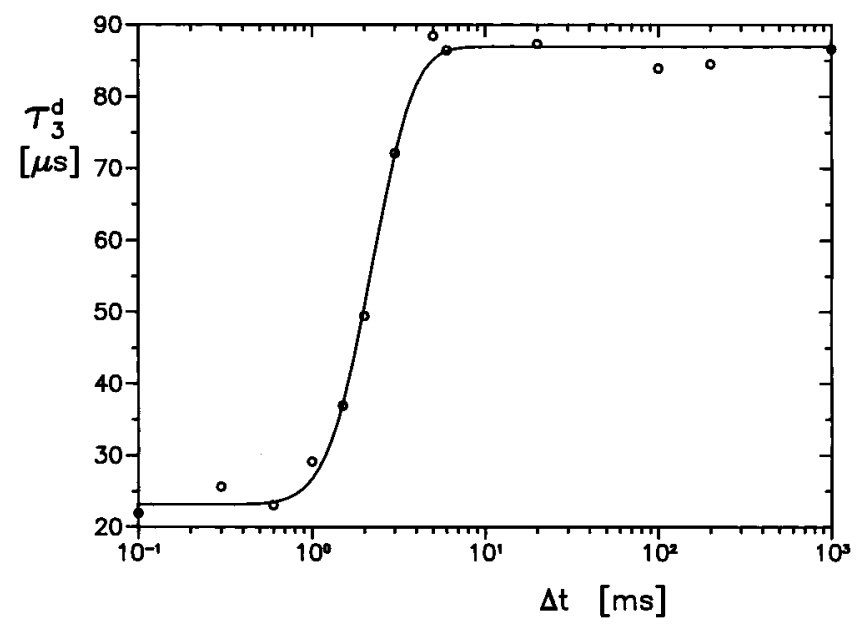

Figure 6. Dichroism decay time constant $\tau_{3, \text { obs }}^{\mathrm{d}}$ of a poly(A) sample with a chain length of 1290 adenylate residues as a function of the time $\Delta t$ after stop of the flow (buffer A, $10{ }^{\circ} \mathrm{C}, 248 \mathrm{~nm}$ ). The line represents a least-squares fit according to the parallel mechanism with the parameters $\tau_{3, \mathrm{k}}^{\mathrm{d}}=23.1 \mu \mathrm{s}, \tau_{3, \mathrm{n}}^{\mathrm{d}}=87 \mu \mathrm{s}, \tau_{\mathrm{p}}=0.9 \mathrm{~ms}$, and $\kappa=7.1$. The time constant measured at $\Delta t=0$ is shown in this plot at $\Delta t=$ 0.1 .

sample with a given chain length is shorter than that of DNA with the same chain length. However, the overall rotational time constant may be taken as a reliable measure of the hydrodynamic dimensions. When these hydrodynamic dimensions are used as scaling parameter, the effects for poly(A) and DNA are very similar. For example, poly(A) of chain length 1290 shows an overall rotational time constant of $87 \mu \mathrm{s}$, which is accelerated to $23.1 \mu$ s directly after the flow is stopped. DNA of chain length 859 bp shows an overall rotational time constant of $60.3 \mu \mathrm{s}$, which is accelerated to $16.7 \mu$ s directly after the flow is stopped. Thus, the similarity is remarkable. A close correspondence is also found for poly(A) of chain length 490 and DNA of chain length $256 \mathrm{bp}$.

It is of particular interest that the time constants for the transition from the flow induced state to the standard state are also very similar for poly(A) and DNA. The stacking reactions of poly(A) are known to be very fast ${ }^{15,16}$ with relaxation time constants around $1 \mu \mathrm{s}$. Thus, it is hardly possible that the time constant $\tau_{\mathrm{p}}=0.9 \mathrm{~ms}$ found for poly(A) is due to a reaction controlled step, e.g., a resealing reaction.

Bacteriorhodopsin: Membrane Protein Disks. The combined results obtained for DNA and poly(A) samples suggest that the special effects are due to turbulent motion of solvent volume elements, left over from the flow phase of the stopped flow experiments. If this explanation holds, similar effects should be observed for polymers of different chemistry and structure. Bacteriorhodopsin was used as a test object because of its stability and its high electrooptical signals. ${ }^{17}$ Bacteriorhodopsin forms thin circular disks ${ }^{18,19}$ with a high permanent dipole moment perpendicular to the plane and a high polarizability parallel to the plane. In the present experiments a relatively high field strength $(15.8 \mathrm{kV} / \mathrm{cm})$ was used for orientation; in this range the orientation is mainly due to the induced dipole moment. ${ }^{17}$

The dichroism decay time constant of the bacteriorhodopsin sample under standard conditions was $65 \mathrm{~ms}$. Exposure to stopped flow experiments induced a dramatic acceleration by more than 3 orders of magnitude to about $15 \mu \mathrm{s}$, when the time constant was measured directly after the flow was stopped. After the flow was stopped the time constants slowly returned to the standard value within a time range of $\sim 500 \mathrm{~ms}$ (cf. Figure 7). 


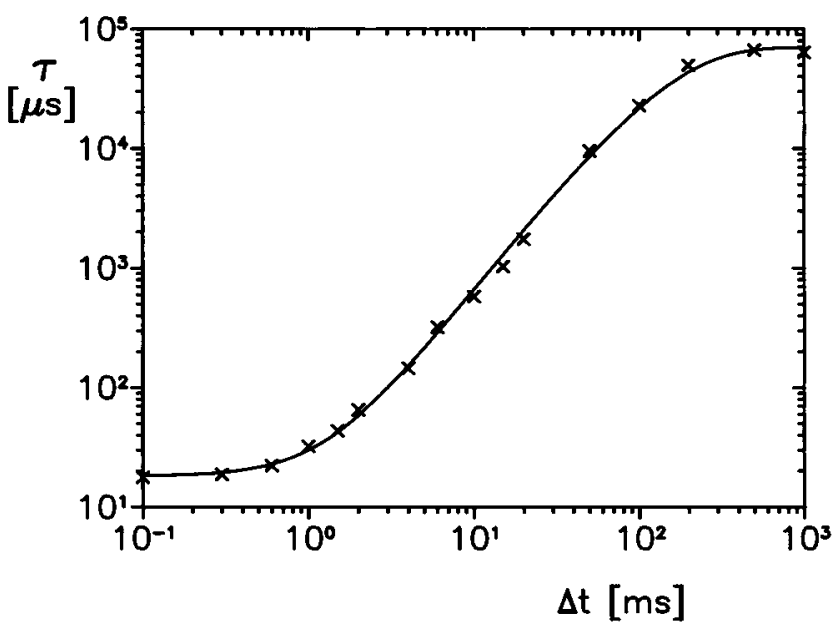

Figure 7. Dichroism decay time constant $\tau_{\mathrm{d}}$ of bacteriorhodopsin as a function of the time $\Delta t$ after stop of the flow (buffer B, $10{ }^{\circ} \mathrm{C}, 546$ $\mathrm{nm})$. The line represents a least squares fit according to the parallel mechanism with the parameters $\tau_{\mathrm{k}}^{\mathrm{d}}=18.1 \mu \mathrm{s}, \tau_{\mathrm{n}}^{\mathrm{d}}=70.7 \mathrm{~ms}, \tau_{\mathrm{p}}=140$ $\mathrm{ms}$, and $\kappa=1.75$. The time constant measured at $\Delta t=0$ is shown in this plot at $\Delta t=0.1$.

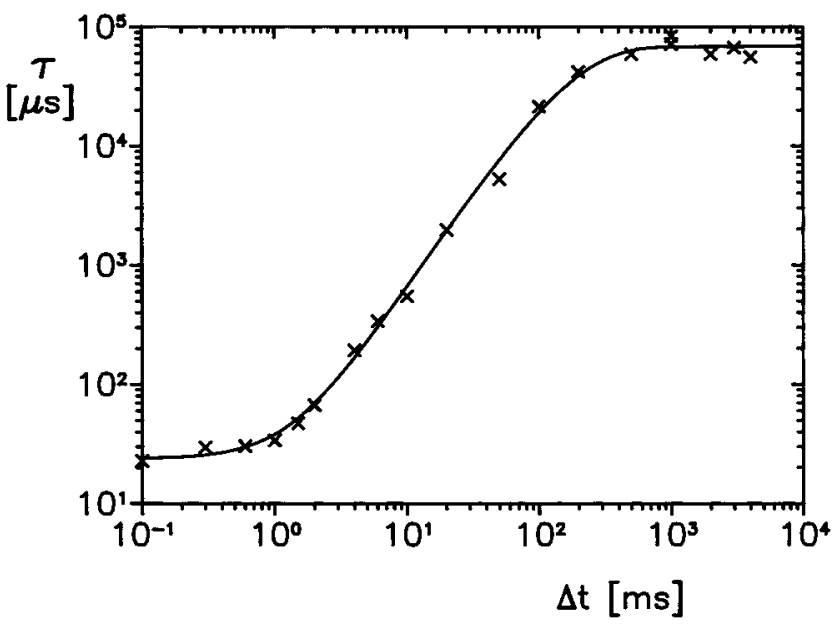

Figure 8. Dichroism decay time constant $\tau_{\mathrm{d}}$ of attapulgite as a function of the time $\Delta t$ after stop of the flow (buffer A, $10^{\circ} \mathrm{C}, 248 \mathrm{~nm}$ ). The line represents a least squares fit according to the parallel mechanism with the parameters $\tau_{\mathrm{k}}^{\mathrm{d}}=23.6 \mu \mathrm{s}, \tau_{\mathrm{n}}^{\mathrm{d}}=68.5 \mathrm{~ms}, \tau_{\mathrm{p}}=162 \mathrm{~ms}$, and $\kappa$ $=1.66$. The time constant measured at $\Delta t=0$ is shown in this plot at $\Delta t=0.1$.

It is remarkable that these data can be fitted at a satisfactory accuracy by the parallel reaction model. The "reaction" time constant obtained from this fit is $162 \mathrm{~ms}$ (cf. Figure 7).

Attapulgite: Suspension of a Silicate Structure. The colloidal particles in suspensions of the clay attapulgite have an "approximate cylindrical form" (Plummer and Jennings ${ }^{20}$ ). Thus, attapulgite suspensions should be very useful for a final comparison, because the chemical structure of attapulgite is very different from the other structures used in the experiments described above. Attapulgite showed very large signals using dichroism detection. The nature of this electric dichroism has not been investigated but was simply used to measure decay time constants. The decay time constant measured without flow was about $70 \mathrm{~ms}$, whereas the decay time constant measured directly after the flow was stopped was $\sim 23 \mu$ s. Thus, the decay is accelerated by more than 3 orders of magnitude. The return from the flow induced state to the standard state takes about $500 \mathrm{~ms}$ (Figure 8). These data can also be fitted at a satisfactory accuracy by the parallel reaction model. The "reaction" time constant obtained from these data is $162 \mathrm{~ms}$.

\section{Discussion}

The development of a stopped flow electric field jump instrument with a high time resolution and a high sensitivity provides a unique tool for the analysis of macromolecular reactions. The quantitative analysis of the state of macromolecules during reactions by electrooptical measurements is a particularly attractive application of this instrument. The electric dichroism and its decay is very sensitive to both global and local elements of the macromolecular structure and its dynamics. The results obtained in the present investigation, however, may not reflect the structure or the dynamics of the macromolecules but rather the structure and the dynamics of the solvent. These possibilities of interpretation are discussed first for the case of nucleic acids.

Because the stopped flow technique has not been used for mixing of reagents in the present experiments, the observed effects must be induced by flow. As described in the Materials and Methods Section, the flow used in the present experiments is characterized by a high Reynolds number and thus is clearly turbulent. Shear stress in turbulent solutions is known to induce various reactions of polymers and of colloids. ${ }^{21}$ For the case of DNA double helices, it is known that shear may induce fracture. ${ }^{22,23}$ Under the conditions of the present experiments the double helices are not fractured, because the length of the double helices is relatively low. Independent experiments demonstrated that long DNA chains, e.g., $\lambda$ DNA, are fractured to segments of about $2000 \mathrm{bp}$ under the stopped flow conditions used in the present investigation (ref 24 and unpublished observations).

Because of the high Reynolds number it is obvious that the flow in the present experiments is turbulent until the stop of flow. However, how fast turbulence decays after the flow is stopped has to be checked. Probably turbulence persists for some time after the flow is stopped and, thus, affects rotational diffusion of the DNA fragments. The rotational diffusion of DNA fragments with 95 and 179 bp remains constant at any time after the flow is stopped, indicating that the solvent conditions after the flow is stopped do not affect their rotational diffusion. This control appears to be evidence for an interpretation of the effects observed for longer DNA fragments by a mechanism involving kinking and resealing of kinks.

However, the problem is more complex. The rotational diffusion of the $95 \mathrm{bp}$ fragment DNA, for example, is particularly fast. Thus, the experiment with this fragment only demonstrates that motion of solvent vortexes is slow compared to rotational motion of this fragment. The rotational diffusion of the fragment with 256 base pairs is already much slower because of its larger hydrodynamic dimensions. Due to its increased dimensions, the motion of this fragment may be influenced by solvent vortices which have no effect on shorter fragments. Thus, it is likely that the rotational diffusion of long fragments is affected by turbulence under conditions where the rotational diffusion of short fragments remains unperturbed.

A strong argument against an interpretation of the present data by kinking is provided of the results obtained for poly(A). As described in the Results Section, the data for poly(A) are in close agreement with those for DNA, when the effective hydrodynamic length is used as a scaling parameter. The correspondence is also very close with respect to the time constant for the backreaction. This time constant of $1 \mathrm{~ms}$ appears to be compatible with a resealing reaction of kinks in DNA double helices, but is hardly compatible with a reaction controlled process in single stranded poly(A), because stacking reactions ${ }^{15,16}$ in single stranded poly(A) are well-known to be very fast (time constants $\leq 1 \mu \mathrm{s}$ ). 
Finally, the data obtained for bacteriorhodopsin and for attapulgite strongly support the conclusion that the rotational motion of particles is strongly accelerated by turbulence left over after the flow is stopped. Because the chemical structure of bacteriorhodopsin and attapulgite is quite different from that of polynucleotides and thus the mechanical properties are expected to be quite different, it is hardly conceivable that kinking of these structures occurs on a corresponding time scale. Both bacteriorhodopsin and attapulgite are of much larger dimensions than the polynucleotides used in the present investigation. Thus, bacteriorhodopsin and attapulgite reflect vortex motions for much longer times than the polynucleotide samples.

The decay of turbulence has been discussed by various authors. In the textbook Gerthsen Physik ${ }^{25}$ an estimate on the lifetime of turbulent motion is based on a simple comparison of kinetic and friction energies. According to this estimate the lifetime of turbulence in the stopped flow observation chamber with a diameter of $2 \mathrm{~mm}$ is $\sim 50 \mathrm{~ms}$ (using the viscosity of water at $10{ }^{\circ} \mathrm{C}$ ). Estimates of the half-lives of a decaying "potential" vortex and of a "Taylor" vortex ${ }^{26-28}$ with a radius corresponding to that of the observation chamber are $\sim 0.5$ and $0.2 \mathrm{~s}$, respectively. The estimated order of magnitude is consistent with the results obtained for both bacteriorhodopsin and attapulgite.

The present results indicate a special sequence of events in the decay of turbulence. As shown by the data obtained for DNA fragments, small vortices with a high rate of rotational motion already decay with a time constant of about $1 \mathrm{~ms}$. Larger vortices, which are reported by objects of larger dimensions, continue to exist until about $100 \mathrm{~ms}$. These data, together with results of simulations reported in the literature, ${ }^{29}$ indicate the following sequence of events: after the flow is stopped there are large numbers of small vortices with high rates of rotational motion, which decay by merging into larger ones with lower rotation rates. This process continues until finally the largest vortices compatible with the dimensions of the observation chamber appear and decay. This broad spectrum of vortexes is reflected in the broad spectrum of dichroism decay time constants found for bacteriorhodopsin and attapulgite.

It is remarkable that the data obtained for compounds of very different chemical structure can all be described with a satisfactory accuracy by the parallel reaction model. It should be noted that the decay time constants obtained for 859 bp DNA, bacteriorhodopsin, and attapulgite immediately after the flow is stopped are surprisingly close to each other, although the time constants obtained under standard conditions are quite different. This result indicates that the mode of acceleration by vortex motion is very similar, whereas the amount of acceleration is quite different. Apparently there is a given structure and dynamics of vortexes immediately after the flow is stopped, resulting in a given decay time constant of molecular orientation. With increasing delay after the flow was stopped, small and fast vortexes merge into larger and slower ones, resulting in larger decay time constants of molecular orientation.

The focus of the present discussion is on the dichroism decay time constants. However, measurements of the electric dichroism also provide electric parameters. An example is given for the case of the 859 bp DNA fragment (Figure 5). The polarizability of the flow induced state is clearly lower than the standard value. This decrease results from the fact that the orientation function used for evaluation does not include the influence of vortex motion. In the presence of vortexes higher electric fields are required to induce a given state of orientational order than in the absence of vortexes: higher electric torques are necessary to align molecules against both thermal and vortex motion. Thus, electric parameters may also be used as a measure of vortex dynamics.

Finally, the potential generation of kinks should be reconsidered. In the presence of turbulence accompanied with high shear stress, generation of kinks in polymer chains or in other macromolecular structures cannot be excluded and, more than that, is quite likely. However, it is hardly possible to distinguish the special effects of vortex motion on dichroism decay curves from those of kinking. Nevertheless, the data obtained in the present investigation indicate that the main effect is due to vortex motion itself, whereas indirect effects resulting from shear stress may provide an additional contribution.

Acknowledgment. The technical assistance of Hartmut Dangendorf in part of the measurements and of Jürgen Wawrzinek in the preparation of solutions is gratefully acknowledged. The facilities of the Gesellschaft für wissenschaftliche Datenverarbeitung $\mathrm{mbH}$, Göttingen, were used for data processing.

\section{References and Notes}

(1) Gomezhens, A.; Perezbendito, D. Anal. Chim. Acta 1991, 242, 147.

(2) Frieden, C.; Hoeltzli, S. D.; Robson, I. J. Protein Sci. 1993, 2, 2007.

(3) Porschke, D. Biophys. J. 1998, 75, 528.

(4) Fredericq, E.; Houssier, C. Electric Dichroism and Electric Birefringence; Clarendon, Oxford, U.K., 1973.

(5) Fresco, J. R.; Doty, P. J. Am. Chem. Soc. 1957, 79, 3928.

(6) Diekmann, S.; Hillen, W.; Morgeneyer, B.; Wells, R. D.; Porschke, D. Biophys. Chem. 1982, 15, 157. 1259.

(7) Diekmann, S.; Jung, M.; Teubner, M. J. Chem. Phys. 1984, 80,

(8) Porschke, D. Biophys. Chem. 1997, 66, 241.

(9) Hagerman, P. J. Annu. Rev. Biophys. Chem. 1988, 17, 265.

(10) Porschke, D. Biophys. Chem. 1991, 40, 169.

(11) Tirado, M. M.; Lopez Martinez, C.; Garcia de la Torre, J. J. Chem. Phys. 1984, 81, 2047.

(12) Hagerman, P. J.; Zimm, B. H. Biopolymers 1981, 20, 1481.

(13) Porschke, D.; Jung, M. J. Biomol. Struct. Dyn. 1985, 2, 1173.

(14) Mills, J. B.; Vacano, E.; Hagerman, P. J. J. Mol. Biol. 1999, 285 , 245.

(15) Porschke, D. Mol. Biol. Biochem. Biophys. 1977, 24, 191.

(16) Porschke, D. Biopolymers 1978, 17, 315.

(17) Porschke, D. Biophys. J. 1996, 71, 3381.

(18) Stoeckenius, W.; Lozier, R. H.; Bogomolni, R. A. Biochim. Biophys. Acta 1979, 505, 215.

(19) Oesterhelt, D.; Bräuchle, C.; Hampp, N. Quart. Rev. Biophys. 1991 24,425 .

(20) Plummer, H.; Jennings, B. R. Brit. J. Appl. Phys. 1968, 1, 1753.

(21) Onuki, A. J. Phys. Condensed Matter 1997, 21, 6119.

(22) Bowman, R. D.; Davidson, N. Biopolymers 1972, 11, 2601.

(23) Reese, H. R.; Zimm, B. H. J. Chem. Phys. 1990, 92, 2650.

(24) Porschke, D. Biochemistry 1984, 23, 4821.

(25) Vogel, H. Gerthsen Physik; Springer: Berlin, 1995.

(26) Lugt, H. J. Introduction to Vortex Theory; Vortex Flow Press: Potomac, MD, 1996.

(27) Albring, W. Elementarvorgänge Fluider Wirbelbewegungen; Akademie Verlag: Berlin, 1981.

(28) Taylor, G. I. Aero. Res. Comm. $R \& M$ 1918, 598.

(29) Watanabe, T.; Iwayama, T.; Fujisaka, H. Phys. Rev. E. 1998, 57, 1636. 